\title{
Myocardial Injury in Patients with COVID-19 in ICU in Marrakech, Morocco: A Restrospective Study
}

\author{
Boumraya Sara $^{1 *}$, Hajar Skali ${ }^{1}$, Bouzari Jihane ${ }^{2}$, Aboulmakarim Siham ${ }^{1}$, Hachimi Abdelhamid ${ }^{2}$ \\ ${ }^{1}$ Department of Biochemistry, E-rrazi Hospital, Mohamed VI Medical Center, Faculty of Medicine and Pharmacy of Marrakech, Cadi \\ Ayyad University, Marrakech, Morocco \\ ${ }^{2}$ Department of Intensive Care Unit, Mohamed VI Medical Center, Faculty of Medicine and Pharmacy of Marrakech, Cadi Ayyad \\ University, Marrakech, Morocco
}

DOI: $10.36348 /$ sjpm.2021.v06i04.005

| Received: 08.02.2021 | Accepted: 20.02.2021 | Published: 22.04.2021

*Corresponding author: Boumraya Sara

\section{Abstract}

The novel coronavirus disease 2019 (COVID-19) has caused an outbreak and become a major public health issue and great concern from international community. Cardiac injuries were suspected and may even be considered as one of the leading causes for death of COVID-19 patients, especially for those with cardiovascular (CV) risk factors. The aim of this study is to investigate if cardiac injury (CI) in COVID-19, preexisting or acquired, is associated to increased mortality in these patients from intensive care unit (ICU) in Mohammed VI hospital in Marrakech, Morocco, from March 20, 2020 to May 20, 2020. Fifty-two patients with laboratory-confirmed COVID-19 were included. Demographic, clinical and laboratory data were extracted from electronic medical records (EMR). 22.7\% patients had CI, all of them developed a HF during COVID-19 course and have died. Among these patients with HF, 44\% had comorbidities (vs $7.3 \%$ of patients without HF $\mathrm{p}<0.05)$. They were older $(61.1$ years old vs 50.5$), 44 \%$ had hypertension and diabetes. Preexisting CV disease was reported in 44\% (vs 1\%). In terms of laboratory findings, Tn-T (med: 231; 422; 10.3; 8.87; 97.28; 41.86; 59.2; 39.27; 68 pg/mL), Nt-proBNP $(553 ; 152 ; 3166 ; 134 ; 306 ; 17900 ; 2524 ; 59000 ; 450$ UI/L), levels of Creactive protein (med: 147 [106.4-240] vs 162 [5-508.3] mg/L) and electrolyte disturbances: hypokalemia (88\% vs $86 \%, \mathrm{p}<0.05)$. Early evaluation and monitoring of cardiac damage with distinct attention to the elderly and highest $\mathrm{CV}$ risk factors may identify patients with HF and contributed to decreased COVID-19 mortality.

Keywords: Covid-19, Myocardial injury, Mechanisms of myocardial injury, Heart failure, Cardiac biomarkers, Covid-19 complications.

Copyright (C) 2021 The Author(s): This is an open-access article distributed under the terms of the Creative Commons Attribution 4.0 International License (CC BY-NC 4.0) which permits unrestricted use, distribution, and reproduction in any medium for non-commercial use provided the original author and source are credited.

\section{INTRODUCTION}

A novel coronavirus in Wuhan has infected clusters in December 2019 and caused an outbreak of severe pneumonia with worldwide human-to-human transmission. As of September 11, 2020, there were 28040853 confirmed cases and 906092 deaths [1, 2]. Primarily, Covid-19 affects the lungs causing interstitial pneumonitis and severe acute respiratory distress syndrome (ARDS). Besides focusing on the severe condition of pneumonia, the novel coronavirus may attack many important organs and cause multiple organ failure with cytokine storm. The heart is one of these most important organs and it is highly suspicious that viral myocarditis and myocardial injury could be also involved and may even be considered as one of the leading causes for the death of COVID-19 patients. However, the evidence is not yet fully determined and the knowledge of this disease is still limited but it is evident that there is an important cardio-vascular (CV) consequence with potential implications for patients with CV risk factor [4]. Moreover, early cases investigating fatality rate reported that cardiac injury (CI) increased the risk of mortality [5]. In fact, abnormal cardiac biomarkers are common on severe Covid-19, reflect of CI, due to a variety of mechanisms that include the Cytokine storm,a high inflammatory burden, inducing myocarditis, and cardiac arrhythmias $[6,7]$. Then the virus entry itself through angiotensinconverting enzyme 2 (ACE2) receptors Covid-19, suggested treatment QTc prolonging and among others. Furthermore, elevated CI markers such us troponin and natriuretic peptides are correlated with perilous course, heart failure (HF) and death [8].

The aim of this study is to investigate whether previous history of CVD, cardiac injury and heart failure, increased the risk of severity and mortality using electronic medical records (EMR) of COVID 19 
positive patients from intensive care unit (ICU) in Mohamed VI hospital in Marrakech, Morocco.

\section{METHODS}

We performed a retrospective observational study, including 52 patients (39 males, 13 females) admitted to the ICU of Mohammed VI hospital in Marrakech, Morocco, from March 20, 2020 to May 20, 2020 and diagnosed with COVID 19 by Real Time Polymerase-Chain Reaction from the nasopharyngeal swab. Severe Covid-19 was defined based on the guidelines of the National Health Commission of Morocco, collaborating with the Moroccan Society of Anesthesia, Analgesia and Critical Care (SMAAR) and the Moroccan Society of Emergency Medicine (SMMU).

The clinical data of patients were collected (age, coronary artery disease (CAD), hypertension, cardiac injury and heart failure as well as necessity of mechanical ventilation and mortality among patients). Cardiovascular disease was defined as any cardiac pathology including the hypertension. Cardiac biomarkers measured on admission were collected, and during hospitalization, including high-sensitivity troponin (hs-TnT) and N-terminal pro-brain natriuretic peptide (NT-proBNP). CI or cardiac involvement was defined mainly as Troponin $\mathrm{T}$ above the 99th percentile limit of upper reference and levels of $\mathrm{N}$-terminal probrain natriuretic peptide (NT-proBNP)>400UI/L with reduced ejection fraction in electrocardiographic defined HF.

At least one echocardiographic evaluation in all patients during hospitalization in ICU is required to assess cardiac function and to explore SARS-Cov myocarditis: segmental wall motion abnormalities, left ventricular ejection fraction $\mathrm{LVEF}<50 \%$, or presence of left ventricular wall thickening $>10 \mathrm{~mm}$ and or pericardial effusion. Electrocardiogram (ECG) changes (ST elevation or ST/T segment changes) are also an element of definition. However, functional and morphological cardiac abnormalities were infrequent.

\section{Statistical Analysis}

The descriptive statistics are median and interquartile range for continuous data. The statistics for categorical variables are counts and percentages. Student's $t$ test and Mann-Whitney U test were performed respectively for normally and non-normally distributed quantitative data. A p value less than 0.05 was regarded as statistically significant. All statistical analyses were performed using IBM SPSS statistics version 16.0 for Windows.

\section{RESULTS}

Fifty two consecutive patients with diagnosis of Covid-19 in ICU were included in the study. According to definition of cardiac injury, 9 of them (22.7\%) had cardiac injury and then developed HF. Patients with HF had higher mortality than those without (all of them [100\%] vs 22 of 43 [51\%] of patients without HF; $\mathrm{p}<0.05)$. Concerning patients with HF, 2 were females and 7 were males $(22.2 \%$ vs $77.8 \%$; $<0.05$ ), had more comorbidities (44\% vs $7.3 \%$; $\mathrm{p}<0.05)$.

Among cardiovascular risk factors, these patients were older (61.1 [53-73] vs 50.5 [31-85] years; $p<0.05$. $44 \%$ of them had hypertension and diabetes, $22 \%$ had renal failure, $33.3 \%$ were former smokers, and $11 \%$ of them were current smoker. Preexisting cardiovascular disease included coronary artery disease (present in 4/9 of patients, $44 \%$ ).

In terms of laboratory findings, high-sensitivity troponin $\mathrm{T}$ (med: $231 ; 422 ; 10.3 ; 8.87 ; 97.28 ; 41.86$; 59.2; $39.27 ; 68 \mathrm{pg} / \mathrm{mL}) \quad$ N-terminal pro-B-type natriuretic peptide(IQR]:553;152; 3166; 134; 306; $17900 ; 2524 ; 59000 ; 450 \mathrm{UI} / \mathrm{L})$, levels of C-reactive protein ( [IQR], 147 [106.4-240] vs 162 [5-508.3] $\mathrm{mg} / \mathrm{L})$.

Procalcitonin done on $3 / 9$ with $\mathrm{CI}(2.33 ; 1.84$; $0.22 \mathrm{ng} / \mathrm{ml}$ vs median of 0.7 in all ICU patients), electrolyte disturbances :hypokalemia (8/9 [88\%] vs 37 of 43 [86\%]; $\mathrm{P}<0.05)$. And all of them had a higher proportion of multiple mottling and ground-glass opacity in radiographic findings .

Greater proportions of patients with HF required invasive mechanical ventilation than those without (7of 9 [77.7\%] vs 16 of 43 [37\%]; P<0.05).

Complications were more common in patients with $\mathrm{CI}$ or HF than those without $\mathrm{CI}$ and included acute kidney injury $(6 / 9$ [66\%] vs 8 of $43[18 \%]$; $<0.05)$. 
Table-1: Demographic characteristics and clinical findings on included patients with COVID-19

\begin{tabular}{|c|c|c|c|}
\hline \multirow{2}{*}{\multicolumn{2}{|c|}{$\begin{array}{l}\text { ICU patients } \\
(\mathrm{n}=52)\end{array}$}} & $\begin{array}{l}\text { Patientswith } \\
\text { cardiacinjury }\end{array}$ & \multirow{2}{*}{$\begin{array}{l}\text { Patients without } \\
\text { cardiac injury } \\
(n=43)\end{array}$} \\
\hline & & $(\mathrm{n}=9)$ & \\
\hline Median (IQR) & No. $(\%)$ & Median (IQR) & Median (IQR) \\
\hline
\end{tabular}

\begin{tabular}{|c|c|c|c|c|c|}
\hline \multicolumn{2}{|l|}{ Age } & \multirow{2}{*}{\begin{tabular}{|l}
$58(\mathbf{2 9 - 8 5})$ \\
39 \\
\end{tabular}} & \multirow{2}{*}{-} & \multirow{2}{*}{\begin{tabular}{|l|l} 
61.1 Y old \\
[53-73]
\end{tabular}} & \multirow{2}{*}{$\begin{array}{l}\mathbf{5 0 . 5} \text { Y old } \\
{[31-85]} \\
32(74 \%) \\
\end{array}$} \\
\hline Gender & Male & & & & \\
\hline & Female & 13 & 26 & $2(22 \%)$ & $11(25 \%)$ \\
\hline \multirow[t]{5}{*}{ Comoribities } & Diabetes & 13 & 25 & $4(44 \%)$ & $9(20 \%)$ \\
\hline & HTA & 13 & 25 & $4(44 \%)$ & $9(20 \%)$ \\
\hline & heart diseases & 4 & 5 & $4(44 \%)$ & $1(2 \%)$ \\
\hline & kidney diseases & 4 & 5 & $2(22 \%)$ & 2 \\
\hline & Tabac & 4 & 7,7 & $1(11 \%)$ & 3 \\
\hline \multicolumn{2}{|c|}{ Patient underrespirator } & 23 & 44,8 & $7 / 9(77 \%)$ & $16 / 43(37 \%)$ \\
\hline \multirow[t]{2}{*}{ TTT Antiviral } & HYDROXYCHLOROQUIN+AZYTHRO & 30 & 78,9 & $100 \%$ & $21(48 \%)$ \\
\hline & Lopinavir+Ritonavir & 6 & 15,8 & $5 / 9(55 \%)$ & 1 \\
\hline
\end{tabular}

\section{DISCUSSION}

The present study demonstrates that a myocardiac injury, as a common complication $(22.7 \%)$, was associated with an unexpected high risk of mortality during hospitalization $(100 \%)$. All $9(22 \%)$ patients with CI developed a HF, defined as elevated natriuretic peptides with left ventricular (LV) dysfunction and reduced ejection fraction in electrocardiographic.

After admission ECG's examination of patients with CI were performed during the periods of elevation of cardiac biomarkers. All ECGs were abnormal, with findings compatible with myocardial ischemia, such T-wave depression and inversion, STsegment depression and Q waves.

According to a recent study of the largest clinical sample in China, $7.2 \%$ of COVID-19 patients developed acute CI. Furthermore, the incidence of CI as reported in China increases with the severity of illness and patients in ICU were more likely to have CI (22.2\%) than non-ICU patients [9]. This observation suggests that $\mathrm{CI}$ is possibly associated with the clinical outcomes of COVID-19 in this population.

Also, the study of She et al found that $19.7 \%$ of patients with COVID-19 had CI, independently associated with an increased risk of mortality in patients with COVID-19 [11].

The first question to ask: How severe acute respiratory syndrome coronavirus 2 (SARS-Cov-2) gives heart damage? And how cardiac involvement could increase morbidity and mortality in these patients with severe COVID-19?

Apparently, there are several possible pathogenic considerations: First, SARS-Cov2 compared to its SARS ancestors have a tropism for CV system, which would explain the occurring of the CV impairment de novo in severe COVID-19 patients or the aggravation of a preexistent cardiopathy in others. Coexistence of COVID-19 may considerably enhance the severity of an underlying CV disease, already proven in some respiratory infections [11-13]. Then, inflammatory cytokine storm or interferon inflammatory response and immune dysregulation T1 and T2 helper cell response, as an important factor in the pathogenesis of vascular disease, induce myocardial interstitial fibrotic response, with deleterious CV effects including atrial and ventricular arrhythmias [78].Therefore, in patients with SARS-CoV-2 infection, underlying $\mathrm{CV}$ disease can aggravate the pneumonia, decrease the systemic oxygenation during pneumonia and increase the severity of symptoms.

Otherwise, several drugs currently used to treat COVID-19 infection, have been shown to have an adverse medication effects [14]: Chloroquine/hydroxychloroquine and azithromycin, block a potassium channel that can prolong the QTc and has been implicated in cases of torsades de pointes (TdP) with risk of arrhythmias and sudden cardiac death, especially when electrolytes imbalance is associated [15]. So conduction defects, ventricular arrhythmias, and HF have been reported with azithromycin therapy [16]. Lopinavir/Ritonavir and interleukin therapies have been shown to be associated with ischemic heart disease and abnormalities in lipid profile [16].

Other proposed mechanism on myocardial injury based on recent studies, incriminates direct myocardial involvement mediated via ACE2. Direct viral myocardial infection might be mediated by ACE2, through (ACE-2) receptors, widely expressed on epithelial and endothelial cells, in the lungs and in the cardiovascular system, SARS-Cov-2 enter cells and, 
ACE2-related signaling pathways might cause a direct insult to myocytes [17].

Last but not the least, the non-specific symptomatology of COVID-19 can lead to misdiagnosis of a possible CI in patients with COVID19 which could delay therapeutic care.

The most common comorbidities among patients admitted to hospitals worldwide have been hypertension and diabetes, followed by cardiovascular and respiratory diseases $[4,6,8]$. Many recent studies have indicated $\mathrm{CV}$ disease as a risk factor for severe COVID-19 [18, 19]. In our study, diabetes and preexisting $\mathrm{CV}$ diseases (hypertension and coronary heart disease) are significantly associated with death in patients with CI than in those without cardiac injury (100\% vs $51 \%$, p<0.05).

Our investigation confirms previous reports of the relationship of older age, underlying $\mathrm{CV}$ disease (coronary artery disease, heart failure, current smoking, and renal failure), with death in patients with cardiac damage in Covid-19. Our results also suggest that women are proportionately more likely than men to survive the infection. In the study of Yang et al, the case mortality rate more than tripled for patients with underlying CV disease [19]. In fact, it is intuitive that the underlying cardiovascular disease may decrease the body's reserve to fight a severe infection, data to confirm.

Various mechanisms have been suggested above to explain the increased vulnerability of patients with underlying CVD for severe COVID-19 disease. However, no direct causative role of such comorbidities to SARS-CoV-2 infection has been shown.

Also, it should be noted that the elevation of troponin seems to have a different prognostic depending on the presence or not of these comorbidities and preexisting coronary heart disease [18]. The elevation of troponin is possibly a reflection of $\mathrm{CV}$ comorbidities.

During hospitalization, the mortality rate increased in association with the magnitude of the reference value of hs-TnT, patients with elevated TnT levels had more frequent arrhythmias (3/9 [33\%] vs $1 / 43$ [2.3\%]), mechanical ventilation (7/9 [77.7\%] vs $16 / 43$ [37\%]) compared with patients with normal TnT levels. The potential explanation for these findings is exposed before, it is altered myocardial demand-supplyratio, increased cardio metabolic demand due to systemic infection coupled with hypoxia caused by acute respiratory illness can impair myocardial oxygen demand lead to acute myocardial injury.
In our study, 9/52 (22\%) of patients exhibiting extreme elevation in Nt-Pro-BNP with rate of death $(100 \%)$, attributed to cardiac failure. They are patients with pre-existing heart disease who presented left ventricular (LV) dysfunction. HF patients are at especially increased risk of morbidity due to their reduced immunity, and reduced hemodynamic ability to cope with severe infections. In the study of Yang et al, the incidence of HF was significantly higher in non survivors compared to survivors [19].

In addition, we observed a manifest hyperinflammatory state results in marked elevations in CRP, cytokines such as interleukin (IL6), high serum ferritin and elevated natriuretic peptides (suggesting cardiac inflammation or dysfunction). Observations suggest a high frequency of cardiovascular events, with patients dying from cardiac arrhythmias and $\mathrm{HF}$, once these biomarkers become established.

In terms of radiologic findings, bilateral pneumonia multiple mottling and ground-glass opacity were more prevalent in patients with than those without CI.

In according to that $\mathrm{Li}$ et al found that patients with acute phase infection of SARS, presented impaired left ventricular performance observed via echocardiography with subsequent $\mathrm{HF}$ and a risk of sudden death in the setting of the structural heart disease itself [17]. Suggesting that mechanisms leading to such cardiac failure are numerous, in common with those mentioned before: Increased viscosity during febrile illnesses, heightened coagulation systems, proinflammatory effects, or endothelial cell dysfunction [20]. Also, viral infections exacerbate a preexisting HF, the parenchymal lung disease then the ARDS, induced by COVID-19 may lead to pulmonary hypertension and subsequent right sided $\mathrm{HF}[21,14]$. In the other hand, both chloroquine and hydroxychloroquine, with Azithromycin are QTc prolonging medications can cause challenging drug-drug interactions and in some cases, increase the risk of QTc prolongation in HF population.

In our study, no previously healthy individuals have a HF as a manifestation of severe COVID-19 infection. In the literature, HF in previously healthy individuals can be due to severe acute myocarditis resulting in cardiogenic shock, which can then result in multi-organ dysfunction syndrome (MODS) and death [22]. Others begun to conjecture about the cardiac contribution to advanced complications in COVID-19 and whether raised filling pressures and a HF phenotype was incriminated. 


\section{CONCLUSION}

COVID-19 infection has been associated with myocardial injury, it may trigger arrhythmias, acute coronary syndromes, and exacerbate underlying heart conditions, which has been implicated with more severe disease courses, HF and even death, data to confirm by larger series. In fact, HF in the setting of COVID-19 is common and may be encountered de novo or in patients with preexisting cardiac disease and can complicate presentation, management and prognosis. The role of biomarkers is capital; it should be used with distinct attention at highest risk population with underlying structural cardiac disease.

Due to the acuteness of this pandemic, the scientific world currently, lacking evidence of physiopathological mechanisms of this myocardial injury. A multidisciplinary approach may optimal for understanding and management of patients with cardiac injury.

Declaration of competing interests: The authors report no relationships that could be construed as a conflict of interest.

\section{REFERENCES}

1. Rothan, H. A., \& Byrareddy, S. N. (2020). The epidemiology and pathogenesis of coronavirus disease (COVID-19) outbreak. Journal of autoimmunity, 109, 102433.

2. John Hopkins Coronavirus Research Centre. https://coronavirus.jhu. edu/map.html. [Accessed 14 May 2020].

3. WHO. (2020). Director-General's opening remarks at the media briefing on COVID-19 - 28 September 2020-[Internet]. Who.int. Available from: https://www.who.int/directorgeneral/speeches/detail/who-director-general-sopening-remarks-at-the-media-briefing-on-covid19---28-september-2020

4. Madjid, M., Safavi-Naeini, P., Solomon, S. D., \& Vardeny, O. (2020). Potential effects of coronaviruses on the cardiovascular system: a review. JAMA cardiology, 5(7), 831-840.

5. Guan, W. J., Ni, Z. Y., Hu, Y., Liang, W. H., Ou, C. Q., He, J. X., ... \& Zhong, N. S. (2020). Clinical characteristics of coronavirus disease 2019 in China. New England journal of medicine, 382(18), 1708-1720.

6. Li, B., Yang, J., Zhao, F., Zhi, L., Wang, X., Liu, L., ... \& Zhao, Y. (2020). Prevalence and impact of cardiovascular metabolic diseases on COVID19 in China. Clinical Research in Cardiology, 109(5), 531-538.

7. Wang, D., Hu, B., Hu, C., Zhu, F., Liu, X., Zhang, J., ... \& Peng, Z. (2020). Clinical characteristics of 138 hospitalized patients with 2019 novel coronavirus-infected pneumonia in Wuhan, China. Jama, 323(11), 1061-1069.
8. Huang, C., Wang, Y., Li, X., Ren, L., Zhao, J., Hu, Y., ... \& Cao, B. (2020). Clinical features of patients infected with 2019 novel coronavirus in Wuhan, China. The lancet, 395(10223), 497-506.

9. Guan, W. J., Ni, Z. Y., Hu, Y., Liang, W. H., Ou, C. Q., He, J. X., ... \& Zhong, N. S. (2020). Clinical characteristics of 2019 novel coronavirus infection in China. MedRxiv. https://www.medrxiv.org/conten t/10.1101/2020.02.06.20020974v1

10. Shi, S., Qin, M., Shen, B., Cai, Y., Liu, T., Yang, F., ... \& Huang, C. (2020). Association of cardiac injury with mortality in hospitalized patients with COVID-19 in Wuhan, China. JAMA cardiology, 5(7), 802-810.

11. Dhainaut, J. F., Claessens, Y. E., Janes, J., \& Nelson, D. R. (2005). Underlying disorders and their impact on the host response to infection. Clinical infectious diseases, 41(Supplement_7), S481-S489.

12. Peiris, J. S. M., Chu, C. M., Cheng, V. C. C., Chan, K. S., Hung, I. F. N., Poon, L. L., ... \& HKU/UCH SARS Study Group. (2003). Clinical progression and viral load in a community outbreak of coronavirus-associated SARS pneumonia: a prospective study. The Lancet, 361(9371), 1767-1772.

13. Viasus, D., Cillóniz, C., Cardozo, C. G., Puerta, P., Garavito, A., \& Torres, A. (2018). Early, short and long-term mortality in community-acquired pneumonia. Ann Res Hosp, 2(5).

14. Driggin, E., Madhavan, M. V., Bikdeli, B., Chuich, T., Laracy, J., Biondi-Zoccai, G., ... \& Parikh, S. A. (2020). Cardiovascular considerations for patients, health care workers, and health systems during the COVID-19 pandemic. Journal of the American College of Cardiology, 75(18), 2352-2371.

15. Goldstein, E. J., Owens Jr, R. C., \& Nolin, T. D. (2006). Antimicrobial-associated QT interval prolongation: pointes of interest. Clinical Infectious Diseases, 43(12), 1603-1611.

16. Limsreng, S., Marcy, O., Ly, S., Ouk, V., Chanroeurn, H., Thavary, S., ... \& Ségéral, O. (2016). Dyslipidemias and elevated cardiovascular risk on lopinavir-based antiretroviral therapy in Cambodia. PLoS One, 11(8), e0160306.

17. Li, S. S. L., Cheng, C. W., Fu, C. L., Chan, Y. H., Lee, M. P., Chan, J. W. M., \& Yiu, S. F. (2003). Left ventricular performance in patients with severe acute respiratory syndrome: a 30-day echocardiographic study. Circulation, 108(15), 1798-1803.

18. Guo, T., Fan, Y., Chen, M., Wu, X., Zhang, L., He, T., ... \& Lu, Z. (2020). Cardiovascular implications of fatal outcomes of patients with coronavirus disease 2019 (COVID-19). JAMA cardiology, 5(7), 811-818. 
19. Yang, J., Zheng, Y., Gou, X., Pu, K., Chen, Z., Guo, Q., ... \& Zhou, Y. (2020). Prevalence of comorbidities in the novel Wuhan coronavirus (COVID-19) infection: a systematic review and meta-analysis. Int J Infect Dis, 10.

20. Nguyen, J. L., Yang, W., Ito, K., Matte, T. D., Shaman, J., \& Kinney, P. L. (2016). Seasonal influenza infections and cardiovascular disease mortality. JAMA cardiology, 1(3), 274-281.

21. Kytömaa, S., Hegde, S., Claggett, B., Udell, J. A., Rosamond, W., Temte, J., ... \& Vardeny, O.
(2019). Association of influenza-like illness activity with hospitalizations for heart failure: the atherosclerosis risk in communities study. JAMA cardiology, 4(4), 363-369.

22. Ruan, Q., Yang, K., Wang, W., Jiang, L., \& Song, J. (2020). Clinical predictors of mortality due to COVID-19 based on an analysis of data of 150 patients from Wuhan, China. Intensive care medicine, 46(5), 846-848. 